\title{
Application of nanosatellites PlanetScope data to monitor crop growth
}

\author{
Svitlana Kokhan $^{1 *}$ and Anatoliy Vostokov ${ }^{1}$ \\ ${ }^{1}$ Department of Geoinformatics and Aerospace Research of the Earth, National University of Life and Environmental Sciences \\ of Ukraine, 17 Vasylkivska St. Kyiv, 03040
}

\begin{abstract}
In this research, an approach to monitor crop growth and development is presented using time series satellite data of high spatial resolution. Monitoring of winter wheat phenology based on images of PlanetScope constellations is considered. By applying various PlanetScope data processing types and ground based GreenSeeker data, differences of NDVI values at two variants of crop fertilization are determined. In particular, the following approaches were used in the research: obtaining the Top of Atmosphere Reflectance (TOA), the Planet Surface Reflectance (SR), and receiving NDVI image in Python using a Rasterio module. It was estimated that NDVI values derived from the surface reflectance imagery were significantly correlated to the ground data of a manual active GreenSeeker optical sensor $(p<0.05)$. The proposed simplified technique, based on PlanetScope NDVI time series, demonstrates the possibilities to monitor temporal changes in crop growth.
\end{abstract}

Keywords: NDVI vegetation indices, PlanetScope constellations, surface reflectance, Top of Atmosphere reflectance

\section{Introduction}

Remote sensing methods provide actual and valuable information about crop growth being non-destructive mean to obtained repeated data from a field. There are advances in the use of remote sensing data because of the application of various sensors and increased their spatial resolution. The challenge is to evaluate the seasonal changes in the various vegetation indices and their correlations with different plant characteristics. Atmospheric conditions may prevent data acquisition from satellite. Such limitations could be taken into account using data from nanosatellite constellations.

\section{Literature review \& problem statement}

Evaluation of the impact of agricultural practices can be increased using per-field remote sensing monitoring both for large-scale areas, and for precision agriculture applications. The perspectives and timely repeatable information on within-field variability in crop growing have specific utility in precision agriculture as a means to optimize production efficiencies via more sustainable and spatially explicit management practices [1-4]. This allows analysing tendencies and monitors conditions of crop growing based on spatio-temporal consistent data of high-frequency time series, identify stress factors and provide more effective and timely information to support local decision-making. As a result, it is possible to provide more accurately financial planning and improve the main indicator of the profitability of agricultural production.
Currently, various remote sensing (RS) data are used to effectively monitor and provide regular feedback on the status and productivity of agricultural fields. However, for their optimal use in precision farming, it is necessary to solve many problems associated with the use of remote sensing technologies: providing simplified access to data, a large number of data processing, using these data to create extended time series, a quick exchange of analytic data with endusers.

Free data of traditional satellites is limited in terms of spatial resolution and image capture frequency. For example, the Landsat- 8 satellite has very good technical characteristics $[5,6]$, but its 16-day survey cycle does not allow obtaining critical significant temporal information at various phases of crop growth and development, which is the main limitation for assessing crop status.

A pair of Sentinel-2 satellites [7] provides spatiotemporal survey frequency with the possibility of reviewing once every five days. But at present, the simultaneous requirements for high spatial $(<10 \mathrm{~m})$ and temporal resolution of the survey are satisfied only by the targeted use of commercial multi-sensor systems, which are limited by weather conditions, deviation of the survey angle from the nadir and being financially expensive.

In the past few years to overcoming the limitations of the spatio-temporal frequency of surveys has been proposed using the PlanetScope nanosatellite constellation of Planet Labs. These nanosatellites 
capture the territory almost in nadir [9], delivering images in four spectral bands with a resolution of 3-4 square meters 3 times a day.

S. Yuval et al. [10] developed a methodology for determining the sowing date on a field scale from PlanetScope images when sowing without plow cultivation. D. Ichikawa et al. [11] developed the operational processing system for Landsat-8, Sentinel2, and PlanetScope satellite data to complex use for monitoring crop growth. The satellite data in these studies were pre-processed and corrected spectral bands were adjusted for the integration of different sensor data. The satellite data in these studies were preprocessed and corrected spectral bands were adjusted for the integration of different sensor data.

A. Tuzcu et al. [12] compared the effectiveness of classification methods on rule-based (RB) classification and Support Vector Machine (SVM) for creating thematic land-use maps based on PlanetScope and WorldView-3 satellite imageries. The classification accuracy of the selected classes according to PlanetScope and WorldView-3 images for the RB classification was $87 \%$ and $94 \%$, and the accuracy of the SVM classification was $93 \%$ and $96 \%$, respectively.

Y. Cheng [13] used PlanetScope imagery to research and analyse the phenology of vegetation in a semi-arid grassland. Based on the constructed time series of NDVI values, four phenological indicators were obtained: the start of the season (SOS), the end of the season (EOS), the maximum value of the vegetation index and the integral vegetation index from the beginning or the end of vegetation season. The work demonstrated the possibility of using PlanetScope images instead of Sentinel-2 and MODIS images.

R. Gurdak et al. [14] evaluated the possibility of monitoring the stages of development of winter wheat based on Sentinel-2 and PlanetScope images. A good correlation was shown between the vegetation indices NDVI (Normalized Difference Vegetation Index), EVI (Enhanced Vegetation Index), SAVI (Soil-Adjusted Vegetation Index) and ground-based Leaf Surface Index (LAI) measurements, and their dependence on the phase of crop development. The interchangeable use of LAI values for Sentinel-2 and PlanetScope data is recommended.

A.B. Baloloy [15] developed models for predicting biomass for mangroves based on the values of the vegetation indices NDVI, SAVI, GNDVI (Green Normalized Difference Vegetation Index) and SR (Simple Ratio) obtained from the Sentinel-2, RapidEye and PlanetScope satellite systems.

M. Gašparović et al. [16] developed a new method for Sentinel-2 and PlanetScope imagery fusion based on an assessment of the accuracy of classification of vegetation cover by NDVI values. The fusion method validation was provided based on the land-cover classification accuracy. The results show better accuracy for PlanetScope images and fusion methods than for Sentinel-2. R. Houborg et al. [17] used a data mining approach to create a regression set based on model rules that link PlanetScope Red-Green-Blue (RGB) data with NDVI calculation for atmosphericcorrected Landsat-8 data for a desert agricultural landscape. Predictability is most optimal $\left(\mathrm{R}^{2}=0.97\right)$ for images almost coinciding in time. The determination coefficient decreases when the time to obtain data increases.

Y. Shi [18] applied a discriminant analysis for the main biophysical parameters based on a set of different vegetation indices calculated using PlanetScope data for two stages of rice vegetative development and substantiated the possibility of creating a map of assessing the crop losses caused by various diseases. N.H. Hoa [19] used eight vegetation indices derived from PlanetScope data to quantify vegetation cover and detect changes. For classification and mapping of the land cover, the OSAVI (Optimized Soil Adjusted Vegetation Index) index is recommended, which turned out to be the most accurate in comparison with other indicators $\left(\mathrm{R}^{2}>0.9\right)$.

The main problem of most studies is the need to use a comparative assessment of the values of vegetation indices derived from PlanetScope imagery and various optical satellite systems that must undergo a mandatory atmospheric correction. This, in turn, requires the availability of qualified data processing specialists, the necessary software and hardware, and a comparatively long time for obtaining the information necessary for business decision-making. Therefore, there are no enough studies comparing different methods of PlanetScope image processing in order to derive vegetation indices. That is why various approaches to processing PlanetScope data are used in the research to obtain time-series NDVI values derived from PlanetScope imagery.

\section{The aim \& objectives of the research}

The aim of this research is to compare approaches to PlanetScope image processing to provide simplified method of obtaining spectral reflectance values in order to derive vegetation indices for evaluating the state of winter wheat based on NDVI time-series data. The key objectives of this investigation are approaches of obtaining spectral reflectance products.

To achieve the aim, the following tasks were set: to use three approaches of image processing to obtaining various spectral reflectance products; to derive NDVI vegetation indices from time series PlanetScope data; to compare satellite NDVI values with GreenSeeker ground data; to determine a simplified method of image processing for obtaining surface reflectance product.

\section{Data processing technique for PlanetScope}

The territory of the Agronomical Experimental Station of the National University of Life and Environmental Sciences of Ukraine was used as a test site. The spatial and ground data was collected from May 6 to June 2, 2018. During the research period, the test plots are represented by winter wheat crop with the repetition of long-term fertilizer application rates (plot 6 has 
$\mathrm{N}_{95} \mathrm{P}_{120} \mathrm{~K}_{120}$ fertilizer rate, and plot 12 - no fertilizers). Experimental plots are represented in Fig. 1.

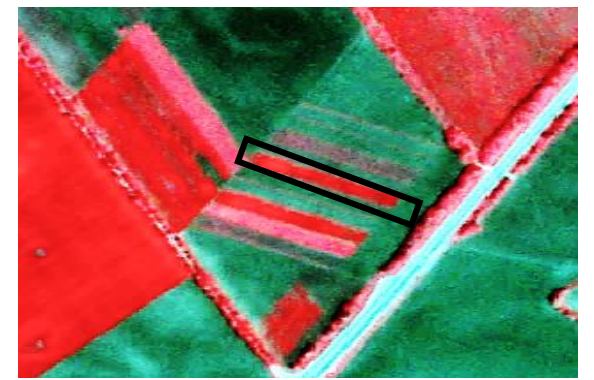

Fig.1. Fragment of PlanetScope image with experimental plots

PlanetScope satellite data captured approximately at the same time of a day (08:24 - 08:25 UTC) with the same spatial resolution $(3,9 \mathrm{~m})$ and viewing angle not exceeding 1.8 degrees. The data is supplied in the metadata.json file.

A PlanetScope nanosatellite property is an inexpensive model of sensor based on CCD-cameras with Bauer's filters. In this regard, the characteristics of PlanetScope equipment do not correspond to the radiometric characteristics, spectral data, and signal-to-noise characteristics of traditional satellite images: the absence of sensor brightness transformations and atmospheric image correction affect consistency on time and space and make difficulties of interpretation for time series data. The characteristics of the spectral bands of PlanetScope are presented in [20].

The standard products, supplied by Planet Labs, are radiometrically calibrated and orthorectified using ground control points for accurate corrections. Digital elevation models for correcting elevation displacement were applied. Removing atmospheric effects increases consistency and comparability between images taken at different times. That is, the obtained values could be used to determine vegetation indices, detect changes in plant development, and create mosaic images [21, 22].

A time-series PlanetScope data is defined as a series of consecutive observations obtained at regular intervals with a spatial resolution of 3-4 $\mathrm{m}$. In our research, two PlanetScope products: 3B_AnalyticMS.tif and 3B_AnalyticMS_SR.tif were used to create a time series of NDVI values for the research area.

A description of the products is presented at the source [22]. 3B_AnalyticMS.tif comes with a scaled brightness that allows us converting 16-bit pixel values to physical units $\left(\mathrm{W} / \mathrm{m}^{2} \cdot \mu \mathrm{m}\right)$. One of the approaches to obtain spectral reflectance product in the research is based on the rasterio module and Python application [23]. It provides simultaneous atmospheric correction of two spectral bands and calculation of the NDVI vegetation index.

The product of surface reflectance 3B_AnalyticMS_SR.tif (SR - Planet Surface Reflectance Product) involves atmospheric correction and allows us to calculate the different vegetation indices. The methodology for obtaining the Planet surface reflectance product is presented in [23].

[Wpisz tekst]
The NDVI vegetation index is considered in the research [24 - 25], since it is an effective and widely used indicator of spatio-temporal changes in assessing the plant growth and productivity. NDVI is calculated by the formula:

$$
N D V I=\frac{\text { Nir-Red }}{\text { Nir }+ \text { Red }}
$$

where Red - Red spectrum and Nir - Near-Infrared spectrum.

Ground measurements were carried out with the GreenSeeker manual active optical sensor, which allows receiving measurements and assessment of plant state, calculating fertilizer application rates and crop forecasts [26]. The principle of operation of the sensor is based on measuring the continuous short radiation of the red and near-infrared spectra. The data obtained are shown on the display as a NDVI vegetative index. The necessary calibration of the device for assessing winter wheat has been carried out. To increase the accuracy of measurements, GreenSeeker data was used taking into account the surface moisture and changes in solar radiation [27]. The general research scheme is presented in Fig. 2.

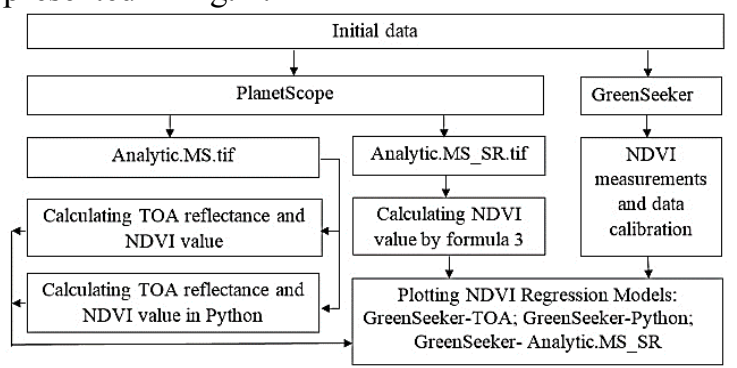

Fig. 2. The general research scheme for a qualitative assessment of the values of atmospheric correction options and the plotting of NDVI regression models

\section{NDVI variability in monitoring of crop growth}

Student's t-Test for independent samples was used to determine the significant/non-significant differences between the NDVI values derived from surface reflectance products using the three approaches to image processing.

For the TOA reflectance option, the $20 \%$ of samples had insignificant differences between the NDVI values of plot 6 (high agricultural background) and plot 12 (absolute control - no fertilizers) at a significance level of 0.95 (Fig. 3a and Fig. 3b).

Calculating spectral reflectance in Python showed, that $23 \%$ of samples had insignificant differences between the NDVI values of two plots, which are differed in the level of agricultural background at a significance level of 0.95 (Fig. 4a and Fig. 4b).

It is supposed to be connected with differcences in data acquisition of PlanetScope and MODIS, which data is based to be used in algorithms of Planet atmospheric correction.

For NDVI values, calculated from the surface reflectance product, $100 \%$ of the samples had a significant difference between the NDVI values of two plots at the significance level of 0.95 (Fig. 5a and Fig. 5b). 
5. Evaluation of PlanetScope data processing types

Correlations between NDVI values derived from spectral reflectance products, such as TOA reflectance, surface reflectance calculated in Python, surface spectral reflectance (SR), and GreenSeeker data, were estimated. Graphs of the correlation between the NDVI values received from GreenSeeker data and TOA reflectance in two spectral spaces are presented in Fig. 6 . The solid line is the best t-functions, dashed lines show confidence interval 0.95 , coefficient of determination $\left(\mathrm{R}^{2}\right)$ is 0.57 . Graphs of the correlation between the GreenSeeker NDVI values and those calculated in Python are in Fig. 7, where $\mathrm{R}^{2}=0.46$. The highest coefficient of determination in the research $\left(\mathrm{R}^{2}\right.$ $=0.79$ ) was received between GreenSeeker NDVI and those, derived from surface reflectance (Fig. 8).
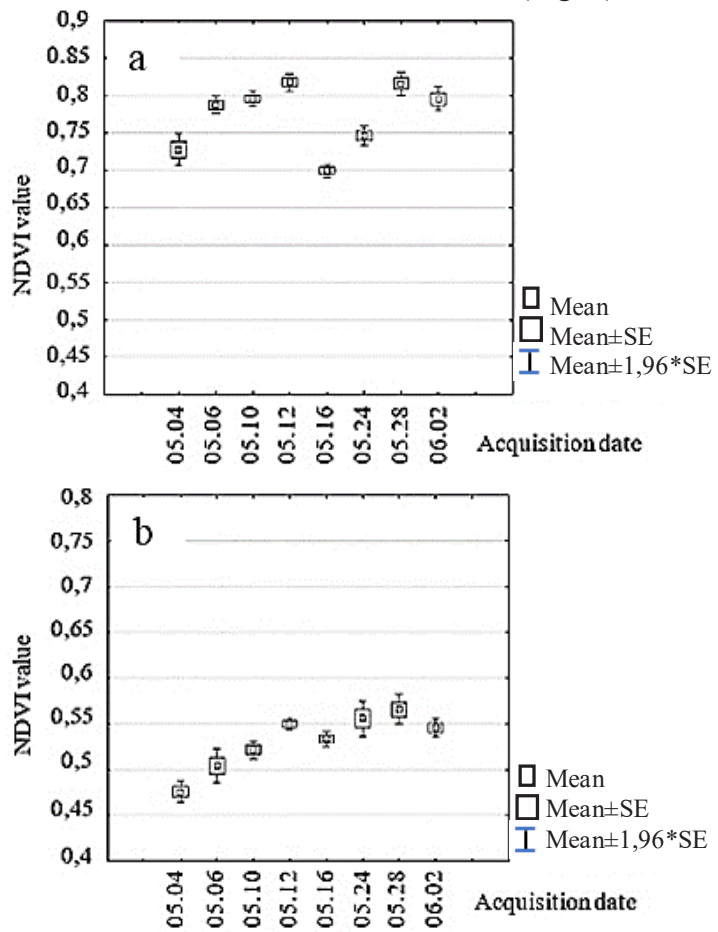

Fig. 3. NDVI values calculated after determination of TOA reflectance: (a) - plot 6 (High Agricultural Background), (b) - plot 12 (Absolute Control - No Fertilizers). SE - standard error

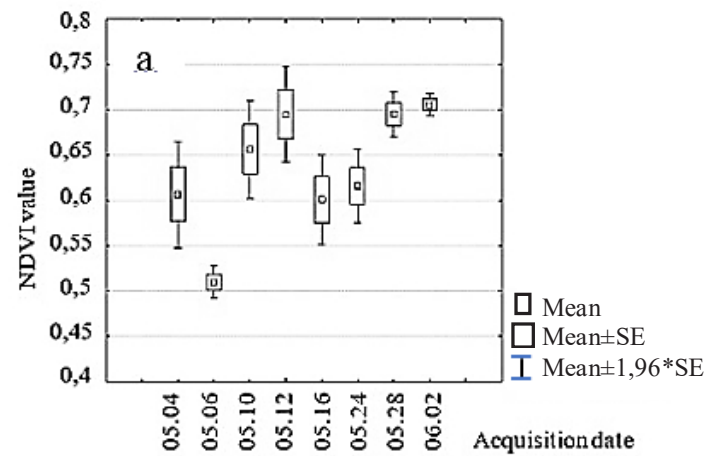

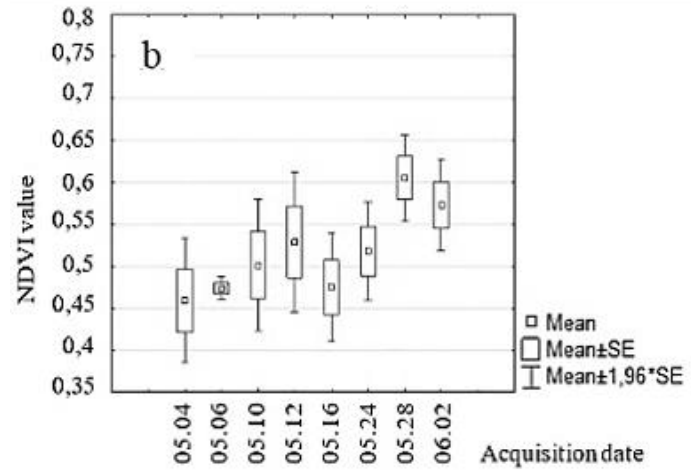

Fig. 4. NDVI values calculated in Python: a - plot 6 (high agricultural background), b - plot 12 (absolute control - no fertilizers)
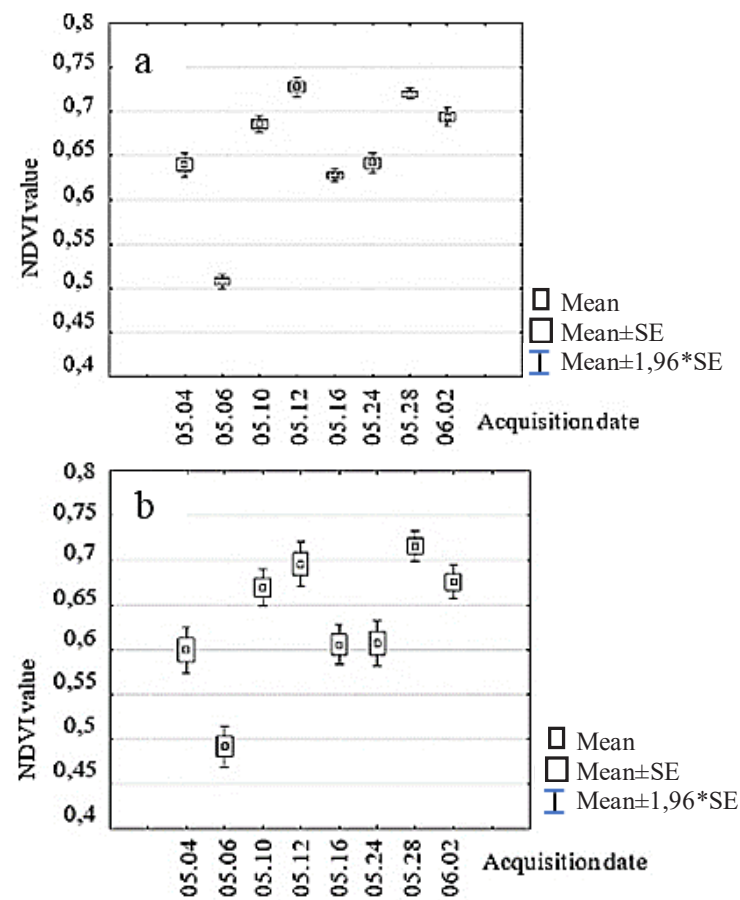

Fig. 5. NDVI values calculated from the surface reflectance product (SR): (a) - plot 6 (High Agricultural Background), (b) - plot 12 (Absolute Control - No Fertilizers)

Based on the results obtained in the studies it can be concluded that generating of an accurate NDVI timeseries, which characterizes the conditions of plant growth and stages of winter wheat development, can be most accurately based on the spectral reflectance

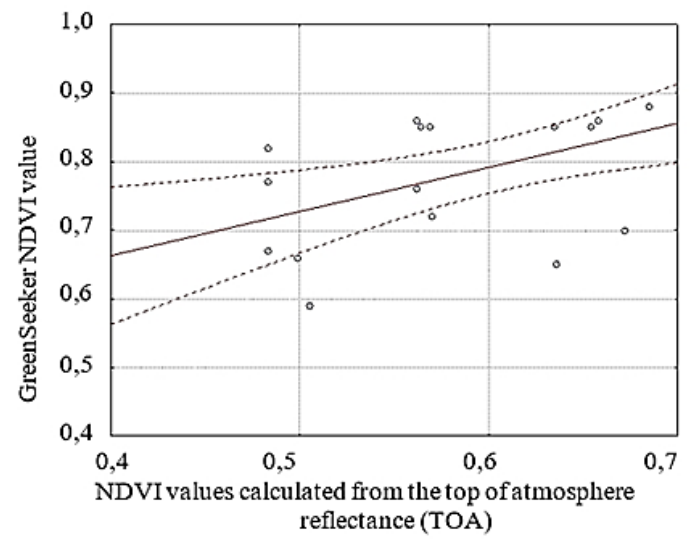


values of 3B_AnalyticMS_SR.tif product and the adjusted GreenSeeker values.

Fig. 6. Graph of the correlation between the NDVI values calculated according to GreenSeeker data and TOA reflectance data in two spectral spaces: $\mathrm{R}^{2}=0.57$. Solid line is the best $\mathrm{t}$-functions, dashed lines show confidence interval 0.95

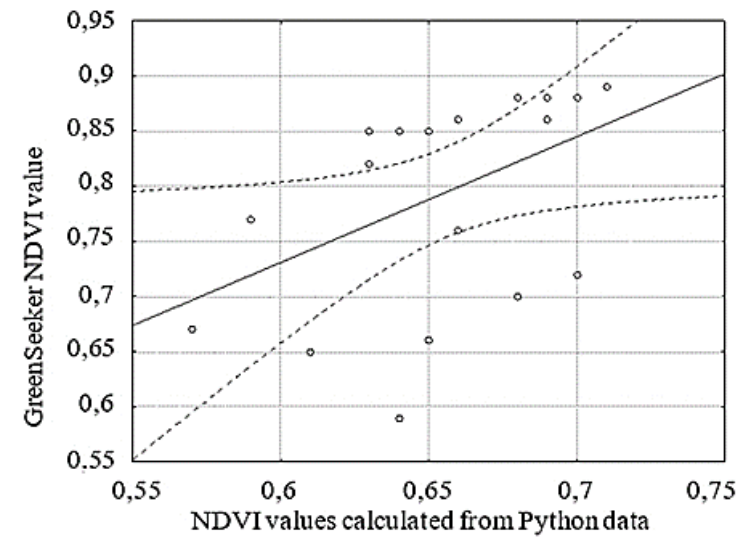

Fig.7. Graph of the correlation between the NDVI values calculated according to GreenSeeker data and Python data in two spectral spaces: $\mathrm{R}^{2}=0.46$. Solid line is the best t-functions, dashed lines show confidence interval 0.95

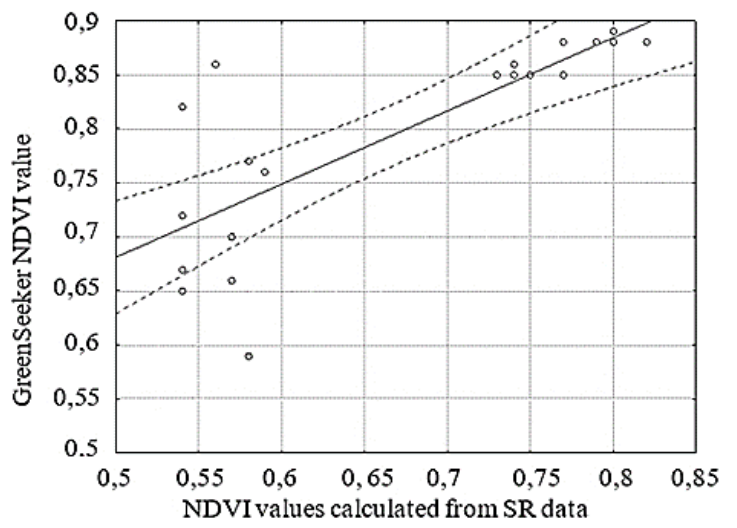

Fig.8. Graph of the correlation between the NDVI values calculated according to GreenSeeker data and $\mathrm{SR}: \mathrm{R}^{2}=0.79$. Solid line is the best $\mathrm{t}$-functions, dashed lines show confidence interval 0.95

\section{Conclusions}

In this research three approaches to PlanetScope image processing were used in order to provide obtaining spectral reflectance values - the Top of Atmosphere Reflectance (TOA), the Planet Surface Reflectance (SR), and receiving NDVI image in Python using the Rasterio module. NDVI time-series values were derived for evaluating the state of winter wheat.

By applying both various PlanetScope data processing approaches, and data of ground sensor GreenSeeker, differences of NDVI values at two levels of crop nutrition were determined. For NDVI values, calculated from the Surface Reflectance product, 100\% of the samples had a significant difference between the NDVI values at the significance level of 0.95 .

Correlations between NDVI values, derived from spectral reflectance products and GreenSeeker data, were estimated. The highest coefficient of determination in the research $\left(\mathrm{R}^{2}=0.79\right)$ was received between GreenSeeker NDVI and those, derived from surface reflectance.

It can be concluded that a simplified method of image processing for obtaining surface reflectance can be most accurately based on the spectral reflectance values of Surface Reflectance product and the adjusted GreenSeeker values.

\section{Acknowledgements}

Publication is funded by the Polish National Agency for Academic Exchange under the International Academic Partnerships Programme from the project „Organization of the 9th International Scientific and Technical Conference entitled Environmental Engineering, Photogrammetry, Geoinformatics Modern Technologies and Development Perspectives".

\section{References:}

1. M.S. Moran, Y. Inoue, Y., E.M. Barnes. Opportunities and limitations for image-based remote sensing in precision crop management. Remote Sens. Envir. 61, 319-346. (1997)

2. N. Zhang, M. Wang, N. Wang. Precision agriculture $-\mathrm{A}$ worldwide overview. Comp. Electr.. Agric. 36(2-3), 113-132. (2002).

3. P.C. Robert. Precision agriculture: A challenge for crop nutrition management. Plant and Soil. 247(1), 143-149. (2002).

4. R. Gebbers, V.I. Adamchuk, V.I. Precision agriculture and food security. Science 327(5967), 828-831. (2010).

5. J.R. Irons, J.L. Dwyer, J.A. BarsiThe next Landsat satellite: The Landsat data continuity mission. Remote Sens. Envir. 122, 11-21. (2012).

6. J. Czapla-Myers, J. McCorkel, N. Anderson, R. Thome, S. Biggar, D. Helder, D. Aaron, L. Leigh, N. Mishra. The ground-based absolute radiometric calibration of Landsat 8 OLI. Remote Sens. 7, 600-626. (2015).

7. M. Drusch, U. Del Bello, S. Carlier, O. Colin, V. Fernandez, F. Gascon, B.Hoersch, C.Isola, P. Laberinti, P. Martimort. Sentinel-2: ESA's optical high-resolution mission for GMES operational services. Remote Sens. Envir. 120, 25-36. (2012).

8. J.W. Rouse, R.H. Haas, J.A. Schell, D.W. Deering. Monitoring vegetation systems in the Great Plains with ERTS. (In 3rd ERTS Symp. NASA SP-351 I, 309-317. 1973). 
9. J. Esper, P.V. Panetta, M. Ryschkewitsch, W. Wiscombe. NASA-GSFC Nano-satellite technology for Earth Science missions. Acta Astronaut, 46 (2-6), 287-296. (2000).

10. S. Yuval, Z.C. Xuan, C. Karine, D.Dunkerley. Sowing date detection at the field scale using CubeSats remote sensing. Comp. \& Electr. Agricul. 157, 568-580. (2019).

11. D. Ichikawa, R. Wakamori. The Integrated Use of Landsat, Sentinel-2 and PlanetScope Satellite Data for Crop Monitoring. (VIGARSS IEEE International Geoscience and Remote Sens. Symposium, 2018).

12. A.G. Tuzcu, N. Taskin, N. Musaoğlu. Comparison of object based machine-learning classifications of PlanetScope and WorldViev-3 satellite images for land usr/cover. (Int. Arch. Photogramm. Remote Sens. Spatial Inf. Sci., XLII-2/W13, 1887-1892. 2019).

13. Y. Cheng. Retrieving vegetation phenology with PlanetScope images for a semi-arid rangeland in Kenia. 1 - 64. (2019).

14. R. Gurdak, P. Grzybowski, P. Feasibility study of vegetation indices derived from Sentinel-2 and PlanetScope satellite images for validating the LAI biophysical parameter to monitoring development stages of winter wheat. Geoinformation, 10, 2735. (2018)

15. A.D. Baloloy, A.C. Blanco, C.G. Candido, R.G.L. Argamosa, C. Dumalag, L.L.C. Dimapilis, E.C. Paringit. Estimation of mangrove forest aboveground biomass using multispectral bands, vegetation indices and biophysical variables derived from optical satellite imageries: RapidEye, PlanetScope and Sentinel-2. (ISPRS Annals of the Photogrammetry, Remote Sens. \& Spatial Inf. Sciences, IV-3, 29-36. 2018).

16. M. Gašparović, D. Medak, I.L. Pilaš Jurjević, I.Balenović. Fusion of Sentinel-2 and PlanetScope Imagery for Vegetation Detection and Monitoring. (Int. Arch. Photogramm. Remote Sens. Spatial Inf. Sci., XLII-1, 155-160. 2018)

17. R. Houborg, M. McCabe M. F. High-Resolution NDVI from Planet's Constellation of Earth Observing Nano-Satellites: A New Data Source for Precision Agriculture. Remote Sens., 8(768), 1-19. (2016).

18. Y. Shi, H. Wenjiang, Y. Huichun, R. Chao, N. Xing, Y. Geng, D.P. Yingying, P. Dailiang. Partial Least Square Discriminant Analysis Based on Normalized Two-Stage Vegetation Indices for Mapping Damage from Rice Diseases Using
PlanetScope Datasets. Sensors, 18(6), 1-16. (2018).

19. N.H. Hoa. Comparison of various spectral indices for estimating mangrove covers using PlanetScope data: A case study in XUAN TYUY National Park, NAM DINH Province. J. of forestry of science and technology, 5, 74-83. (2017)

20. Planet Labs, Inc. Radiometric Calibration of the Planet Labs PlanetScope Constellation. (2018).

21. Patt S.G. Chavez. Image-Based Atmospheric Corrections - Revisited and Improved. Photogram. Eng. \& Remote Sens. 62(9), 1025-1036. (1996).

22. Planet Labs, Inc. Planet imagery product specification: PlanetScope \& RapidEye, 32. (2018).

23. N. Weisiger, J.R. Kyle, D. Bauer. Deriving a vegetation index from PlanetScope imagery. Join GitHub. (2018)

24. A. Collison, N. Wilson. PLANET SURFACE REFLECTANCE PRODUCT. Planet Labs, Inc, 10. (2018).

25. S. S. Kokhan. Applacation of vegetation indexes derived from satellite images IRS-1D LISS-III for determonation of crop status. Space Sciences \& technology. 17(5), 58-63 (in Ukraine, 2011)

26. S.S. Kokhan, S.S. Application of multispectral remotely sensed imagery in agriculture. (ISPRS Technical Commission VII Symposium. 100 Years ISPRS. Advancing Remote Sensing Science. The International Archive of the Photogrammetry, Remote Sensing and Spatial Information Sciences. Vol. XXXVIII. Part 7B. 337-341. 2010).

27. GreenSeeker handheld crop sensor.

28. Y. Kim, D.m. Glenn, j. Park, H.K. Ngugi, B.L. Lehman. Characteristics of active spectral sensor for plant sensing. (American Society of Agricultural and Biological Engineers ISSN 21510032 293. 55(1), 293-301. 2012). 